\title{
Pobreza, desigualdade e eqüidade em saúde: considerações a partir de uma perspectiva de gênero transversal
}

\author{
Poverty, inequality, and equity in health: \\ considerations based on a transversal \\ gender perspective
}

Karen Giffin 1

\footnotetext{
1 Departamento de Ciências Sociais, Escola Nacional de Saúde Pública, Fundação Oswaldo Cruz. Rua Leopoldo Bulhões 1480, Rio de Janeiro, $R J$ 21041-210, Brasil. karengi@ensp.fiocruz.br
}

\begin{abstract}
This article discusses the modernization of gender inequalities which has occurred in Brazil in the last 20 years under the hegemony of neo-liberal macro-economic policies. A concept of gender as transversal is applied to questions of reproductive health (contraception and abortion, prenatal care and birthing, adolescent pregnancy, maternal and reproductive mortality, STIS/AIDS, and violence, among others), permitting analysis by both gender and social class. The history of the PAISM (Program for Integral Health Care for Women), on the other hand, reveals the complex articulation of this national public health program which, although strongly influenced by the Brazilian women's movement, has been infiltrated by heterogeneous interests in the international context. PAISM serves as an example of the appropriation of proposals and principles that were generated by this social movement, but re-articulated to gloss over the process of deepening gender and class inequality.
\end{abstract}

Key words Reproductive Health; Women's Health; Equity; Gender Relations; Gender

Resumo Este trabalho aborda a atualização das desigualdades de gênero que ocorrem no Brasil nas últimas duas décadas, sob a hegemonia das políticas macroeconômicas de cunho neoliberal. No centro desta análise, um conceito de gênero transversal é aplicado a questões da saúde reprodutiva (contracepção e aborto, parto e pré-natal, gravidez na adolescência, mortalidade materna e reprodutiva, DST/AIDS e violência, entre outras) permitindo relacionar os gêneros e comparar mulheres de diferentes classes sociais. A história do PAISM (Programa de Atenção Integral à Saúde da Mulher), por outro lado, revela a complexa articulação entre uma política pública nacional que foi fortemente influenciada pelo movimento de mulheres, mas permeável a interesses heterogêneos no contexto internacional. Serve como exemplo da apropriação e esvaziamento de propostas e princípios advindos deste movimento social, mas rearticulados para encobrir o aprofundamento das desigualdades de classe e de gênero.

Palavras-chave Saúde Reprodutiva; Saúde da Mulher; Eqüidade; Relações entre Gêneros; Gênero 


\section{Introdução: direitos (não) reprodutivos}

A luta do feminismo dos anos 60 partiu da meta de igualdade na diferença sexual. Reivindicando a não-hierarquização das especificidades de homens e mulheres, almejou uma igualdade social que reconhece as diferenças, hoje expressa na noção de "eqüidade de gênero".

Na divisão do mundo em esferas "generificadas", a "divisão sexual do trabalho" que o feminismo questionou, assentou-se a identidade masculina no seu atributo de provedor, e a feminina no seu papel de doméstica e reprodutiva, de mãe. Em termos de sexualidade, esperava-se o exercício da sexualidade masculina desde cedo, fora do âmbito familiar/reprodutivo, enquanto a feminina foi limitada à reprodução de filhos legítimos.

Enquanto vertentes minoritárias do feminismo insistiram na contextualização sóciohistórica de variações nos significados do masculino e do feminino, e apontaram diferenças importantes entre mulheres de diferentes classes sociais, o impulso político maior do movimento revelou, e repudiou, a definição hegemônica da mulher como reprodutora, confinada à esfera do lar: vulnerável, subjugada, excluída, "escrava da espécie” (Beauvoir, 1953).

A reivindicação de "direitos reprodutivos" evoluiu deste entendimento, de que controlar a fecundidade seria uma condição essencial na luta pela igualdade social dos gêneros nestas sociedades, onde a esfera privada, da família, foi feminilizada e desvalorizada, enquanto os valores dominantes eram referidos à atuação no mundo público.

O corpo reprodutivo feminino teria de ser controlado, tanto em nome de uma sexualidade mais igualitária, livre da ameaça da gravidez não-desejada, como para ensaiar condições mais eqüitativas para concorrer na esfera pública da política e do trabalho remunerado. A bandeira feminista "nosso corpo nós pertence", ponto nevrálgico de uma estratégia de transformação das relações de gênero, tanto na esfera privada como na pública, também expressou uma nova consciência do corpo feminino colonizado por outros interesses - incluindo, em primeiro plano, a ciência médica.

Na luta de quase 40 anos pelos "direitos reprodutivos", foram enfatizadas as necessidades de controle da fecundidade: $\mathrm{o}$ direito à contracepção e ao aborto legal. Foram reivindicadas, em primeiro plano, condições de controlar o corpo para evitar a reprodução, recusando a definição hegemônica da identidade feminina e sua redução à maternidade.
Do ponto de vista da eqüidade de gênero, as especificidades das mulheres são todas relacionadas com diferenças sexuais que, elaboradas no social, irradiam desde as vivências sexuais e reprodutivas mais íntimas, até a arquitetura de redutos do poder como congressos nacionais onde não há provisão de banheiros femininos, passando por identidades pessoais, símbolos culturais, normas e instituições sociais, leis, etc. No entanto, como nos lembra Almeida (2000), eqüidade se refere não a toda diferença, mas a diferenças que são consideradas injustas; ou seja, a identificação de ineqüidades é baseada em valores.

Foram priorizados, neste movimento, os espaços e valores públicos a serem conquistados, e os direitos de não reproduzir, sob o entendimento de que o direito de reproduzir estava "dado" (para não dizer "imposto") na ideologia hegemônica (Ávila \& Gouveia, 1996). Com isso, foi deixado em segundo plano a análise das condições concretas para exercitar a maternidade, altamente diferenciadas entre grupos sociais, apesar da hegemônica homogenização ideológica das mulheres.

A história dos "direitos (não) reprodutivos" mostra a internacionalização desta questão, tanto na evolução de redes feministas como nas agências que, desde os anos 60 , promovem o controle populacional como condição dos planos de "desenvolvimento internacional", sob o argumento de que a diminuição da pobreza dos países periféricos dependia desse controle. A partir de 1975, declarado pela Organização das Nações Unidas (ONU) "Ano Internacional da Mulher", as redes feministas são convidadas a ocupar espaço crescente na articulação de definições, declarações e documentos internacionais sobre estes direitos.

Os resultados dos programas de controle populacional em termos de "prevenção de nascimentos" em países periféricos são consideráveis, e em 1990, havia estimativas de que 412 milhões de nascimentos no mundo "em desenvolvimento" tinham sido evitados (Jejeebhoy, 1990). No entanto, não se pode dizer a mesma coisa com respeito à diminuição da pobreza, dada como justificação original destas intervenções. Ao contrário, a distância entre os "direitos" celebrados nos documentos internacionais, e a situação de pobreza aguda da maioria das mulheres no mundo, tem aumentado continuamente neste período. Da perspectiva da sua meta global enunciada, a imensa rede mundial de programas de "desenvolvimento com controle populacional” é um exemplo fulminante de fracasso de políticas internacionais dirigidas à diminuição da pobreza. 
No entanto, este fato recebeu pouca atenção na Conferência Mundial de População e Desenvolvimento no Cairo em 1994. Nesse evento, a introdução da questão da saúde reprodutiva foi celebrada como importante avanço, particularmente dos/as que vinham do âmbito dos estudos demográficos que acompanharam os programas de controle internacionais, até então ocupados exclusivamente com a diminuição das taxas de fecundidade. Da perspectiva da situação vital das populações periféricas, por outro lado, é preciso indagar se enfocar a saúde neste âmbito não representa um cerceamento da atenção, uma forma de evitar discussão das causas da pobreza e vulnerabilidade crescentes, que acompanham estes programas durante quase 40 anos, condicionando a qualidade de vida e saúde no planeta.

\section{Tendências atuais nas condições sociais da reprodução humana: a atualização das desigualdades de gênero no Brasil}

No Brasil, em um contexto sócio-histórico caracterizado pela concentração extrema da renda, acompanhado da dependência externa, a queda brusca da fecundidade e a entrada maciça de mulheres casadas e com filhos na força de trabalho nas últimas décadas (C. Bruschini, 1993, em apresentação feita durante o XVII Encontro Anual da ANPOCS), expressam uma relativização da divisão sexual de trabalho anterior. Mas, embora essas estratégias tenham ajudado na sobrevivência de parcelas majoritárias da população, não lhes permitiram sair da pobreza. E, nas famílias mais pobres, novas contradições são criadas entre a necessidade da renda da mãe e a de cuidados dos filhos pequenos, e entre a necessidade da renda de filhos menores e os estudos, necessários se não suficientes na luta por um futuro, se possível, melhor.

Dados mais recentes de áreas urbanas de baixa renda no Brasil, mostram que com a dificuldade crescente dos homens garantirem uma renda familiar adequada, ou mesmo manter um trabalho minimamente estável, estamos passando por uma "transição de gênero" em que as mulheres não somente "ajudam" como também começam a ser responsabilizadas, e a se considerarem responsáveis, pela provisão de renda, mesmo (ou, principalmente) tendo filhos menores. (Giffin, 1994a; Luz, 1987; Scott, 1990; Simões-Barbosa, 2001). Essa atualização ideológica dos gêneros, na figura da "nova mulher independente" que controla sua fecundidade, trabalha fora e tem seu dinheiro "pró- prio", permite o ocultamento do aprofundamento da dupla jornada, da exploração e da forma em que estas estratégias contribuem para a reprodução da desigualdade em nível de gênero e de classe social.

Dado que os trabalhos remunerados aos quais a maioria das mulheres têm acesso são, em geral, relacionados às suas habilidades "domésticas”, desvalorizadas e mal-pagas (Brito, 2000), sua "dupla jornada" não é fonte de independência financeira e nem mesmo de estabilidade familiar. Ao contrário, embora necessária à sobrevivência e manutenção dos filhos, é intimamente relacionada à desestruturação do provedor masculino no contexto de desemprego e salários inadequados à manutenção de uma família. Este "fracasso" masculino pode resultar em "comportamentos masculinos de desistência, pânico e fuga” (Agier, 1990), ou mesmo na "expulsão" do homem do lar (Scott, 1990).

Apesar do desejo e de repetidas tentativas de "constituir família", as "chefiadas por mulheres” representaram $20 \%$ das famílias brasileiras em 1996. A exacerbação da fragmentação dos laços familiares em condições de pobreza é evidenciada em dados regionais: $21 \%$ das crianças abaixo de 15 anos na Região Sul e $34 \%$ na Região Nordeste, vivem em famílias em que falta pelo menos um dos pais (BEMFAM, 1996).

Quanto mais vulnerável a família, mais importante tem sido sua extensão por meio de relações de compadrio, vizinhança, etc. Na ausência de políticas públicas voltadas para a seguridade social, essas "redes de reciprocidade" entre famílias de baixa renda na América Latina foram apontadas, nos anos 70, como elemento essencial nas suas estratégias de sobrevivência (Lomnitz, 1975). No entanto, não há solidariedade entre os pobres que produza empregos e salários suficientes para resolver a sobrevivência de todos, e muito menos para proporcionar saídas da pobreza. Desde então, com a crescente concentração da riqueza e empobrecimento de segmentos majoritários, estudiosos da família alertam para o fato de que, quanto maior a pobreza e a necessidade dessas redes solidárias, tanto menor os recursos que eles manejam (Jelin, 1994).

É claro que a mulher da classe média também entrou na força de trabalho, em condições e salários melhores, o que obviamente não quer dizer em igualdade com os homens da sua classe. No que diz respeito às suas condições para sair da esfera doméstica, essas mulheres se beneficiam da farta oferta de trabalhadoras domésticas que assumem uma dupla jornada doméstica em troca de salários irrisórios, e à margem das leis trabalhistas. Segundo o De- 
partamento Intersindical de Estatística e Estudos Socioeconômicos (DIEESE, 1998, apud Brito, 2000) em 1995, as trabalhadoras domésticas representavam cerca de $30 \%$ das assalariadas no Brasil, e 2,7\% têm registro em carteira. Esses arranjos entre mulheres permitem à mulher empregadora trabalhar fora não somente por valorizar a realização profissional ou a participação na vida pública, mas também para garantir suas vantagens relativas como classe, mantendo seus filhos fora do mercado de trabalho e pagando escolas e cursos, entre outros recursos de capital cultural, em nome de futuras oportunidades de emprego.

Brito (2000) aponta que a "flexibilização" do trabalho mais praticada atualmente é a da precarização do emprego e do desemprego. Esta reorganização internacional do capital, que tira vantagens dos baixos salários e da fraca regulamentação do trabalho nos países periféricos, tende a transformar as mulheres em força de trabalho preferido. Nessa situação, "a terceirização ao nível internacional corresponde a processo de replicação da dominação das mulheres, pois o que é exportado e explorado é o caráter tradicionalmente desvalorizado do trabalho feminino" (Brito, 2000:198). Ou seja, a velha divisão sexual do trabalho atravessa a nova reestruturação produtiva, e reforça a feminilização da pobreza. Segundo Brito (2000), atualmente, $70 \%$ dos 1,3 bilhão de pessoas que vivem em condições de pobreza no mundo são mulheres.

O fato da "feminilização" da pobreza acompanhar a "feminilização" da força de trabalho, revela os limites da expectativa feminista de que controlar a fecundidade e "conquistar" o mundo público do emprego remunerado trariam nova liberdade para "as mulheres". A atualização das vulnerabilidades dos gêneros, reformuladas, serve ainda para mostrar a impossibilidade de conseguir uma divisão do trabalho doméstico mais igual entre os gêneros sem uma reorganização geral do emprego remunerado de homens e mulheres.

Além disso, enquanto o modelo macroeconômico continua a privilegiar o lucro das multinacionais acima das necessidades das populações, haverá cada vez mais excluídos, cada vez mais vulneráveis - homens, mulheres, crianças e idosos, que sobrevivem ainda interlaçados, neste tecido social em processo de esgarçamento.

\section{O Programa de Atenção Integral à Saúde da Mulher (PAISM) e a saúde reprodutiva das mulheres no Brasil: bases para uma política de eqüidade}

Da perspectiva da luta pela atenção integral à saúde da mulher no Brasil, a nova ênfase na "saúde reprodutiva" nos circuitos internacionais representa uma diminuição de expectativas e um retrocesso conceitual, na medida em que opera, mais uma vez, uma redução (A. M. Costa, 1999). Definido em 1983, em resposta a pressões para a instalação de um programa de controle da fecundidade no país, o PAISM rearticulou esta demanda no interior de uma proposta de atenção integral à saúde das mulheres, recusando e deslocando o limite das funções reprodutivas. Nascido no seio do movimento sanitário nacional pré-constituinte, o PAISM avançou no processo de formulação de um programa de saúde pública integral e eqüitativa como direito universal, a política que este movimento defendia.

Adotando esses princípios, e beneficiadas pelo clima de mobilização política vigente, as formuladoras do PAISM conseguiram promover amplo debate público com profissionais de saúde e integrantes do movimento de mulheres, que desde então lutam pela implementação deste programa oficial em âmbito nacional, a partir de experiências locais (Costa \& Aquino, 2000). O caso de São Paulo em 1988, ilustra os limites dos esforços locais de profissionais dedicado/as: nesse ano, estimativa-se que, das 1.797 unidades de saúde que haviam implementado algum aspecto desse programa, apenas $7 \%$ ofereceram algum método contraceptivo (Hardy, 1988).

Em termos gerais, o abandono do PAISM, no que diz respeito à sua efetiva implementação em âmbito nacional, consolidou uma separação entre o uso de métodos artificiais de controle da fecundidade e os cuidados à saúde para as mulheres mais pobres que dependem dos serviços públicos, que são a maioria no país (Giffin, 1994b).

Tenho caracterizado o processo de controle da fecundidade no Brasil como um caso de "modernidade perversa" (Giffin, 1992). Enquanto a taxa nacional de uso e os métodos usados são "modernos", a "perversidade" é conseqüência do fato de que as escolhas reprodutivas são altamente condicionadas pela pobreza e falta de cidadania. Nessa situação, as condições de controle da fecundidade assim como as condições do parto, são perversas.

Investigações locais das práticas contraceptivas de mulheres de baixa renda mostra- 
ram a pílula sendo tomada irregularmente e/ou incorretamente, comprada nas farmácias sem receita médica, em dosagens inadequadas, e usada durante muito anos seguidos, inclusive por mulheres com problemas cardiovasculares, fumantes, etc. (Costa et al., 1990; Giffin \& Costa, 1995; Wawer et al., 1986). E isso, em um país que ainda em 1974, assinou a Declaração de Bucharest, assumindo como sua a responsabilidade de provisão dos métodos contraceptivos.

Além dos efeitos nocivos à saúde, o uso descontrolado da pílula também resulta em gravidez indesejada e aborto. Há estimativas de que $30 \%$ das gestações terminam em aborto (ilegal) no Brasil (AGI, 1994). Junto com a alta porcentagem de mulheres que admitem ter provocado o aborto, estudos de populações locais também mostraram altos índices de abortos repetidos e malsucedidos (S. H. Costa, 1999; Martins et al., 1991; Valladares et al., 1987). Desde 1940, aborto legal é "garantido" pela lei brasileira no caso de estupro ou risco de vida, mas ainda são rarríssimos os serviços públicos que o realizam.

A esterilização cirúrgica, método de controle da fecundidade mais utilizado no país, até recentemente não era reconhecida oficialmente, nem regulamentada, supervisionada ou registrada. A clandestinidade dessa prática também dificulta pesquisas sobre efeitos colaterais (Molina, 1999). Normalmente, tem sido realizado no momento do parto 4 entre 5 esterilizações, segundo a Sociedade Civil Bem-Estar Familiar no Brasil (BEMFAM, 1996), através da cesárea desnecessária, sendo esta uma das razões do aumento vertiginoso dessa prática no país, que tem uma das taxas mais altas do mundo. A perversidade no controle da fecundidade vai refletir em morbidade e mortalidade materna e reprodutiva, na vulnerabilidade aumentada ao HIV, ao câncer ginecológico e a outras doenças, incluindo hipertensão e infecções do trato reprodutivo (Beral, 1979). No entanto, não há dados sobre a magnitude das infecções do trato reprodutivo no país (Faúndes \& Tanaka, 1992), conformando o que tem sido chamado de "armadilha da desinformação", que expressa a relação de dupla via entre falta de informação e negligência de questões de saúde das mulheres (Graham \& Campbell, 1992).

Até a mortalidade materna é subestimada em aproximadamente 50\% no Brasil. Em 1995, a taxa corrigida estava em torno de 134 mortes/100 mil nascidos vivos (MS, 1995), o que representa $6 \%$ da mortalidade de mulheres entre 10 a 49 anos. Essa taxa, considerada por alguns um indicador geral de qualidade de vida de um país, é muito mais alta no Brasil do que em outros países com desenvolvimento econômico semelhante. Há indícios de aumento (Silva et al., 1999), apesar do fato do governo brasileiro ter assumido compromissos e assinado documentos internacionais se comprometendo a reduzi-la.

Desde os anos 80 , há no país comitês de mortes maternas, e o óbito materno foi declarado de notificação compulsória em 1997. As causas principais dessas mortes são hipertensão, hemorragias, complicações do aborto (ilegal) e infecções puerperais. A taxa em mães solteiras é quase o dobro da de mães casadas, e mais alta nas regiões mais pobres do país (Jácome, 2001). As deficiências da atenção ao parto incluem o número inadequado de leitos. Isso, combinado à falta de transporte, resulta na peregrinação de gestantes na hora do parto, em busca de uma vaga (Valladares, 1999). A maioria dessas mortes seria evitável por meio de uma atenção pré-natal adequada.

As deficiências do pré-natal são refletidas no fato de que, no período entre 1991-1996, $14 \%$ das gestantes de nascidos vivos não tiveram nenhuma assistência pré-natal, $36 \%$ não fizeram vacinação antitetânica e $72 \%$ não tiveram nenhuma consulta pós-parto (BEMFAM, 1996). No que diz respeito à qualidade da atenção, a relação médico-paciente ainda é alvo de preocupação, e infelizmente, não se tem notícias de uma melhoria com a "feminilização" da profissão médica nas últimas décadas.

O modelo de parto tecnológico, medicalizado, predomina no país, cuja taxa de partos cesáreos, 36\% em 1996, é uma das mais altas do mundo (BEMFAM, 1996). Essa prática iatrogênica consolidou-se, embora constituindo um risco à saúde: o coeficiente de mortalidade materna em 1997, era de 52,3 para partos cesáreos e 25,7 para partos normais (Tanaka, 2000). Seu mal-uso é indicado pelo fato das taxas de parto cesáreo serem maiores em hospitais privados e em regiões e grupos de maior renda, ou seja, de gestantes em melhores condições sociais e de saúde (Barros et al., 1996; Rattner, 1996). Em 1998, o Ministério da Saúde (MS) lançou uma portaria que estabelece um limite de pagamento desses partos para cada hospital, o que tem resultado em suspeitas de falsificação de dados (Lannes, 1999). Em 1995, 25\% das cesárias pagas pelo SUS foram de adolescentes.

A taxa de fecundidade específica das adolescentes de 15 a 19 anos aumentou $26 \%$ entre 1970-1991, segundo dados do IBGE (1991). Em 1995, os partos de mães de 10 a 19 anos representaram $22 \%$ dos partos do Sistema de Informações de Nascidos Vivos (SINASC), sendo a 
primeira causa de internação na rede pública (RNFS, 1999). Em 1996, 18\% das jovens de 1519 anos já tinham engravidado (excluindo as que abortaram), com taxas mais altas no Norte e Centro-Oeste e nas áreas rurais. No grupo sem escolaridade, $54 \%$ das jovens já tinham um filho, comparado com $34 \%$ das com até 3 anos de estudo, e 6,4\% das com 9-11 anos (Ferraz \& Ferreira, 1998). Segundo as mães, $49 \%$ desses filhos não foram planejados. Apenas $14 \%$ das jovens de 15-19 anos declararam usar algum método contraceptivo, e curetagens pós-aborto representaram $22 \%$ dos procedimentos do SUS para esse grupo etário em 1997 (RNFS, 1999). Em 1996, os jovens entre 10 a 24 anos de ambos os sexos representaram $13 \%$ dos casos de AIDS, sendo a maior causa de contaminação dos jovens o uso de drogas injetáveis e das jovens as relações heterossexuais (RNFS, 1999).

No que diz respeito às diferenças de mortalidade entre homens e mulheres, estas ainda têm, apesar de tudo, uma expectativa de vida maior. Enquanto as novas tendências de mortalidade entre mulheres em idade reprodutiva refletem o crescimento da AIDS e da violência (Reis et al., 2001), a mortalidade masculina continua a registrar alta nos coeficientes de mortes violentas. No período entre 1980-1995, por exemplo, a mortalidade de jovens masculinos entre 15-19 anos por armas de fogo cresceu em média $9,1 \%$ ao ano; entre os de 20 a 24 anos, esse crescimento foi de $7 \%$ (Szwarcwald \& Leal, 1998).

As questões de eqüidade colocadas nesse campo da ótica do gênero feminino, conjugam especificidades referentes à escassez e dificuldades de acesso de mulheres a serviços de saúde, mas também ao exagerado controle e medicalização do corpo feminino (Costa \& Aquino, 2000). São incluídas questões de: barreiras concretas resultantes da sobrecarga de trabalho e falta de tempo para se cuidar, reforçadas ideologicamente pela definição da mulher como "cuidadora de outros"; o efeito de discriminação e preconceito no que diz respeito - especialmente mas não somente - à sexualidade feminina, que dificulta tanto o acesso quanto a adequada atenção e prevenção das ISTs (infecções sexualmente transmissíveis); exclusão de mulheres em posições hierarquicamente superiores de decisão política, e como integrantes de protocolos de pesquisas básicas em função das complexidades do corpo reprodutivo; inadequado desenvolvimento de métodos de controle da fecundidade masculina, e de métodos femininos de proteção às ISTs; questões da qualidade de serviços que envolvem deficiências quantitativas e qualitativas; entre outras
(Giffin \& Lowndes, 1999; Meloni, 1999; Silver, 1999; Valladares, 1999).

Nos novos estudos do gênero masculino, por outro lado, os homens estão argumentando que os serviços de saúde estão exageradamente feminilizados, tanto em termos do gênero dos profissionais como em serviços, programas e clientela, denunciando que não têm espaço nem horário para atenção à sua saúde (Lopes et al., 2001). Já questionam também a conformação patriarcal da divisão sexual do trabalho, da identidade masculina e da hierarquia entre os gêneros, concluindo, por exemplo, que "dominar ensurdece emoções, sentimentos, e encontros... e diminui o espaço humano e as potências de prazer" (Ribeiro, 1991:36). O que tem sido colocado em termos de "imposição ao gênero feminino” por algumas, é agora retraduzido por outros em "afastamento do gênero masculino" - da possibilidade de convivência com os filhos, das decisões reprodutivas, etc. (Giffin \& Cavalcanti, 1999).

A entrada dos homens nos estudos de gênero acompanha a relativização do poder do patriarca, reduzido a um (pretenso) vendedor da sua força de trabalho individual. Na análise de Nolasco $(1988,1995)$, por exemplo, o homem sofre atualmente uma "dupla violência": do trabalho alienante e informatizado que gera instabilidade no emprego e insegurança, e do individualismo extremo que fragmenta as relações interpessoais. Nolasco relaciona as reações e relações violentas masculinas com o desemprego e com a resultante desestruturação da identidade masculina, que refletem violentamente nas suas parceiras. Nestas condições, uma gravidez pode representar não uma afirmação, mas uma ameaça à virilidade masculina, e estudos apontam que a violência doméstica freqüentemente ocorre durante a gravidez (Meniccuci \& Vianna, 1993). Para Castelo-Branco (1985), o poder do macho na sociedade contemporânea "foi deslocado da rua para a casa, e depois para o corpo", processo no qual o "reino genital" passou a ser uma "usina de poder" e as relações sexuais passaram de uma problemática "da relação" para uma problemática "da carne".

A insatisfação dos homens, que impulsionou a abertura do campo de gênero para a ótica masculina, também pode ser considerada um indicador de dificuldades crescentes em nível de necessidades humanas, sejam de sobrevivência ou sentimentais, de homens e mulheres nestas sociedades.

Vale ressaltar que essas necessidades agora refletem, para a maioria das mulheres, o acúmulo dos efeitos da reprodução, do controle da 
reprodução e do trabalho doméstico acrescentado ao trabalho remunerado, explorado em condições de aguda precarização. E apesar das deficiências dos dados nacionais e dos alardeados "avanços" nos direitos, a saúde reprodutiva é um campo em que as diferenças entre mulheres de diferentes classes sociais mostram-se agudas e, na medida em que os serviços públicos de saúde não atendem às necessidades modernizadas, crescentes.

Este campo ilustra, portanto, a necessidade de enfocar o gênero como relacional e transversal (Kergoat, 1996; Saffioti, 1992), interativo com classe social, raça/etnia, diferenças de geração, capital cultural, etc. e não como uma condição que determina, por si só, diferenciais de vulnerabilidade. Apesar da transformação das práticas e das ideologias dominantes, que oferecem uma nova "homogenização positiva" da "condição feminina" atualmente (Giffin, 1994a), o gênero é um sistema entre outros que atuam de forma interlaçada no plano social, com resultados às vezes contraditórios, diferentes para mulheres (e homens) em variadas situações. A atual feminilização e pauperização da AIDS é emblemática disso, no sentido de ilustrar como as vulnerabilidades do gênero feminino não podem ser abstraídas das vulnerabilidades resultantes da pobreza. Ao mesmo tempo, tanto a AIDS como a violência urbana mostram como a vulnerabilização de alguns membros de uma sociedade pode, com o tempo, atingir a todos.

\section{Eqüidade de gênero e políticas públicas: qual o Estado que queremos?}

“Formular a pergunta sobre a possibilidade de realização de princípios de eqüidade no contexto atual de globalização econômica é interrogar-se sobre o papel do Estado..." (Almeida, 2000:61).

Tanto a situação de saúde reprodutiva das mulheres brasileiras como a condição orçamentária do PAISM indicam, atualmente, os reais limites da luta política restrita ao setor saúde, seja no plano nacional ou no palco dos discursos internacionais.

O caso do PAISM no Brasil, abarcou tudo que as recomendações mais atuais preconizam em termos de desenvolvimento de políticas e programas setoriais eqüitativos em termos de gênero: mobilização e participação da sociedade civil organizada; sensibilização das autoridades e profissionais de saúde; mulheres em posições oficiais de comando; definição de um programa oficial federal com perspectiva de gênero, elaborado com a colaboração ativa da sociedade civil; e novas leis constitucionais. Além disso, mais de dez anos depois, nas Conferências Internacionais do Cairo e Beijing, este programa foi consagrado como proposta avançada, e capitalizado politicamente, pelo mesmo governo cujas mulheres (e homens) vivem as situações acima descritas, e cujo orçamento nem mais o inclui como rubrica.

Se os programas internacionais de controle da fecundidade são um exemplo de fracasso em termos da sua meta anunciada de diminuição da pobreza, a política da saúde reprodutiva no Brasil é um caso paradigmático, que revela aspectos da atuação de um tipo de Estado ainda não plenamente conceitualizado. Nossa lacuna conceitual, neste caso, é: como chamar um Estado que não respeita suas próprias leis? Como chamar as pessoas que vivem neste Estado?

Como coloca Pitanguy (1999:27), na queda brusca da fecundidade que ocorreu no Brasil na real ausência dos serviços públicos preconizados oficialmente no PAISM, "refletiu-se mais o impacto das politicas populacionais vigentes de fato no país do que os princípios feministas já incorporados em leis, normas e programas governamentais". Na conceituação de Penha (1991), as distorções históricas deste processo revelam um "Estado de duas caras" que manteve uma postura liberal de não-interferência ao mesmo tempo em que favorecia a atuação de entidades privadas nacionais e internacionais.

Hartmann conclui que nos anos 90, a linguagem de direitos das mulheres foi apropriada pelos organismos e agências internacionais envolvidos neste campo, onde a questão de gênero "tem se tornado uma forma de as agências populacionais evitarem questões politicamente sensíveis de classe, raça e desigualdades entre países desenvolvidos e não-desenvolvidos" (Hartman, 1995:134). Urbaneja observa que o enfoque de gênero está sendo defendido atualmente pelos mesmos governos que, se subjugando aos mandos neoliberais, diminuem, degradam e desmontam os serviços públicos dos quais os/as mais vulneráveis dependem (Urbaneja, 2000). No processo de desarticulação de organizações representativas e de classe, e sua substituição por negociações e acordos locais e pontuais, “....uno de los actores privilegiados por los gobiernos y las agencias financieras son las mujeres que, bajo la presión de la angustiante problemática cotidiana de sus famílias, intercambian lealdad política por bienes mínimos que refuerza su sumisión a la discriminación de gênero..." (Laurell, 2000:45).

Donna Haraway, preocupada com a relação entre os modelos econômicos que empobrecem 
e excluem as maiorias, e a realidade produtiva e reprodutiva das mulheres pobres, caracteriza a luta feminista no campo da saúde reprodutiva a partir da imagem de um "espéculum virtual na nova ordem mundial” (Haraway, 1997). No argumento dela, o feminismo, ao assumir uma militância na área reprodutiva, se apossou de um "espéculum virtual", um poder de discurso que não resistiu ao poder das novas tecnologias médicas, algumas reprodutivas. Para além do continuado controle da reprodução e do avanço da medicalização do corpo feminino, que são favorecidos pelas tecnologias informatizadas, Haraway chama a atenção pelo fato da produção destas novas tecnologias ser realizada às custas de mulheres trabalhadoras superexploradas nas periferias da nova ordem mundial.

A conclusão emergente é de que tem havido uma cooptação do discurso e um desgaste das energias feministas (Feghali, 2000), e que pronunciamentos oficiais sobre a importância de gênero fazem parte integrante das políticas macroeconômicas hegemônicas que aumentam a desigualdade entre mulheres. Isto equivale a dizer que está havendo um esvaziamento de conceitos e uma crescente ideologização, na qual a visibilização do gênero encobre tanto o aprofundamento das desigualdades sociais como os conflitos de interesse entre mulheres de classes sociais diferentes.

Atualmente, no processo de globalização das políticas neoliberais, os Estados latinoamericanos, incluindo o brasileiro, abrem mão de sua soberania em determinar as macropolíticas econômicas em nome da acumulação do capital externo, ao mesmo tempo em que a bandeira dos "recursos escassos" internos é alçada à única preocupação legítima, e definida em termos estritamente técnicos (Almeida, 2000).

No seu papel ativo de desmonte de si mesmo, das instituições públicas de bem-estar social, esse tipo de Estado suprime a noção de justiça e direitos sociais ou de cidadania social e, como analisa Laurell (2000:42), “sólo puede ser comprendida en el marco de su oposición al Estado de bienestar". Este Estado tem “implementado um Estado de mal-estar social...não tem tomado medidas efetivas contra o desem- prego, não desenvolve políticas sociais condizentes com as necessidades que temos, tem 'leiloado' patrimônios da pátria desnecessariamente e a preços vis e, como é de domínio público, tem dirigido seus esforços para adequar-se ao que as elites nacionais e os organismos financeiros internacionais têm exigido...são gerentes de uma concepção ideológica de Estado antipovo, a chamada 'nova ordem mundial'" (Feghali, 2000:280).

Obviamente, essa concepção de Estado é o oposto dos princípios de eqüidade, integralidade e qualidade que o Movimento Sanitário da América Latina defendeu (Tajer, 2000), já que a acumulação e a eqüidade "se excluem como valores" (Almeida, 2000).

Não é de se surpreender, portanto, que nestes circuitos as idéias de liberdade, igualdade e direitos sejam, hoje, ambíguas (Laurell, 2000), e a noção de eqüidade restrita aos mais necessitados, em vez de expressar um conceito inclusivo de cidadania e justiça social (Almeida, 2000).

Na sua opção de dependência extrema do capital externo, esses Estados são extremamente vulneráveis; e isto requer, por outro lado, que mantenham uma "soberania interna" alta, impedindo a intervenção crítica da sociedade civil (Almeida, 2000; Laurell, 2000). Em outras palavras, “...en esa lógica no figura como sujeto sustantivo la sociedad civil, ésta se concibe mas bien como 'objeto a cooptar, controlar y disciplinar”' (Matamala, 2000:268).

Nossa análise indica que, em resposta à tarefa colocada de delinear diferentes situações que exacerbam ou atenuam a carência material que pode modificar substancialmente a circunstância de precariedade e as perspectivas de solução, somos obrigados a apontar para nós, sujeitos a estes Estados vulneráveis. Boaventura dos Santos refere como "fascismo do Estado paralelo" as práticas destes Estados, "caracterizadas pela grande discrepância entre o direito escrito e a ação estatal prática" (Santos, 1998:34). Nessa situação, a resolução de problemas de pobreza e eqüidade não pode ser concebida apenas em termos setoriais, sem um pacto político amplo, capaz de reivindicar mudanças na política econômica e na forma de a conduzir. 


\section{Referências}

AGI (Alan Guttmacher Institute), 1994. Aborto Clandestino: Uma Realidade Latino-Americana. New York/Washington, DC: AGI.

AGIER, M., 1990. O sexo da pobreza: Homens, mulheres e famílias numa "avenida" em Salvador da Bahia. Tempo Social, 2:35-60.

ALMEIDA, C., 2000. Saúde nas reformas contemporâneas. In: Saúde Eqüidade e Gênero: Um Desafio para as Políticas Públicas (A. M. Costa, E. Merchán-Hamann \& D. Tajer, org.), pp. 61-94, Brasília: ABRASCO/Asociación Latinoamericana de Medicina Social/Universidade de Brasília.

ÁVILA, M. B. \& GOUVEIA, T., 1996. Notas sobre direitos reprodutivos e direitos sociais. In: Sexualidades Brasileiras (R. Parker \& R. Barbosa, org.), pp. 160-172, Rio de Janeiro: Relume-Dumará.

BARROS, F. C.; VICTORA, C. G. \& MORRIS, S., 1996. Caesarean sections in Brazil. Lancet, 346:839-843 BEAUVOIR, S., 1953. The Second Sex. New York: Knopf. BEMFAM (Sociedade Civil Bem-Estar Familiar no Brasil), 1996. Pesquisa Nacional de Demografia e Saúde. Rio de Janeiro: BEMFAM.

BERAL, V., 1979. Reproductive mortality. BMJ, 15:632.

BRITO, J., 2000. Enfoque de gênero e relação saúde/ trabalho no contexto da reestruturação produtiva e precarização da pobreza. Cadernos de Saúde Pública, 16:195-204.

CASTELLO-BRANCO, J. C., 1985. O Manequim de Carne: Imprensa Masculina e a Produção da "Macheza". Dissertação de Mestrado, Rio de Janeiro: Universidade Federal do Rio de Janeiro.

COSTA, A. M., 1999. Desenvolvimento e implementação do PAISM no Brasil. In: Questões da Saúde Reprodutiva (K. Giffin \& S. H. Costa, org.), pp. 319-336, Rio de Janeiro: Editora Fiocruz.

COSTA, A. M. \& AQUINO, E. L., 2000. Saúde da mulher na reforma sanitária brasileira. In: Saúde, Eqüidade e Gênero: Um Desafio para as Políticas Públicas (A. M. Costa, E. Merchán-Hamann \& D. Tajer, org.), pp. 181-202, Brasília: ABRASCO/Asociación Latinoamericana de Medicina Social/ Universidade de Brasília.

COSTA, S. H., 1999. Aborto provocado: A dimensão do problema e a transformação da prática. In: Questões da Saúde Reprodutiva (K. Giffin \& S. H. Costa, org.), pp. 163-186, Rio de Janeiro: Editora Fiocruz.

COSTA, S. H.; MARTINS, I.; PINTO, C. \& FREITAS, S., 1990. Family planning practices in low-income women in Rio de Janeiro, 1984-85. International Family Planning Perspectives, 16:16-22.

FAÚNDES, A. \& TANAKA, A. C., 1992. Reproductive tract infections in Brazil: Solutions in a difficult economic climate. In: Reproductive Tract Infections: Global Impact And Priorities For Women's Reproductive Health (G. Greer, ed.), pp. 253-274, New York: Plenum Press.

FEGHALI, J., 2000. Gênero e controle social na saúde: A omissão dos governos e os limites do monitoramento. In Saúde, Eqüidade e Gênero: Um Desafio para as Políticas Públicas (A. M. Costa, E. Merchán-Hamann \& D. Tajer, org.), pp. 275-296, Brasília: ABRASCO/Asociación Latinoamericna de Medicina Social/Universidade de Brasília.
FERRAZ, A. \& FERREIRA, I., 1998. Início da atividade sexual e características da população adolescente que engravida. In: Seminário Gravidez na Adolescência (E. Meloni, M. E. Fernandes, P. Bailey \& A. McKay, org.), pp. 47-56, Rio de Janeiro: Associação Saúde da Família.

GIFFIN, K., 1992. A modernidade perversa e a reprodução humana no Brasil. In: Saúde, Ambiente e Desenvolvimento (M. C. Leal, P. C. Sabrosa, R. H. Rodrigues \& P. M. Buss, org.), pp. 99-122, Rio de Janeiro: ABRASCO/São Paulo: Editora Hucitec.

GIFFIN, K., 1994a. Esfera de reprodução em uma visão masculina: Considerações sobre a articulação da produção e da reprodução, de classe e de gênero. Physis - Revista de Saúde Coletiva, 4:23-40.

GIFFIN, K., 1994b. Women's health and the privatization of fertility control in Brazil. Social Science and Medicine, 39:355-360.

GIFFIN, K. \& CAVALCANTI, C., 1999. Homens e reprodução. Estudos Feministas, 7:53-71.

GIFFIN, K. \& COSTA, S. H., 1995. As práticas contraceptivas e o aborto no Brasil. In: Reflexões sobre Gênero e Fecundidade no Brasil (Family Health International, org.), pp. 54-75, São Paulo: Family Health International.

GIFFIN, K. \& LOWNDES, C. M., 1999. Gender, sexuality and the prevention of sexually-transmissible diseases: A Brazilian study of clinical practice. Social Science and Medicine, 48:283-292.

GRAHAM, W. \& CAMPBELL, O., 1992. Maternal health and the measurement trap. Social Science and Medicine, 35:967-977.

HARAWAY, D., 1997. The virtual speculum in the new world order. Feminist Review, 55:22-72.

HARDY, E., 1988. Avaliação do PAISM no Estado de S. Paulo. Campinas: Centro de Pesquisas MaternoInfantis de Campinas. (mimeo.)

HARTMANN, B., 1995. Reproductive Rights and Wrongs: The Global Politics of Population Control. Boston: South End Press.

IBGE (Fundação Instituto Brasileiro de Geografia e Estatística), 1991. Censo Demográfico 1991. Rio de Janeiro: IBGE.

JÁCOME, M., 2001. As mulheres e o exercício dos direitos reprodutivos: $\mathrm{O}$ caso da mortalidade materna. Jornal Fêmea, 9:6-7.

JEJEEBHOY, S., 1990. Famplan: The great debate abates. International Family Planning Perspectives, 16:139-142.

JELIN, E., 1994. Las familias en América Latina. Edições das Mulheres, 20:75-106

KERGOAT, D., 1996. Relações sociais de sexo e divisão sexual do trabalho. In: Gênero e Saúde (M. J. Lopes, D. Meyer \&V. Waldaw, org.), pp. 19-27, Porto Alegre: Artes Médicas.

LANNES, R., 1999. Secretaria de Assistência à Saúde restringe as cesarianas. Tema, 17:22-23.

LAURELL, A. C., 2000. Globalización e reforma del Estado. In: Saúde Eqüidade e Gênero: Um Desafio para as Políticas Públicas (A. M. Costa, E. Merchán-Hamann \& D. Tajer, org.), pp. 35-60, Brasília: ABRASCO/Asociacón Latinoamericana de Medicina Social/Universidade de Brasília.

LOMNITZ, L., 1975. Cómo Sobreviven los Margina- 
dos? México, DF: Siglo Ventiuno.

LOPES, A.; AMORIM, A.; COUTINHO, A.; COSTA, C.; OLIVEIRA, G.; GARCIA, J.; BARROS, J.; SILVA, J.; AGUIAR, J.; MOREIRA, L.; BARBOSA, M.; STROZENBERG, P.; SOUZA, R.; ALVES, S.; SILVA, S.; GONÇALVES, V. \& CORREIA, W., 2001. Palavra de Homem. Rio de Janeiro: Núcleo de Estudos em Saúde Coletiva, Universidade Federal do Rio de Janeiro/Escola Nacional de Saúde Pública, Fundação Oswaldo Cruz.

LUZ, M., 1987. Identidade masculina-feminina na sociedade brasileira atual: Crise nas representações. In: Homem, Mulher: Abordagens Psicoanaliticas e Sociais (C. de Poian, org.), pp. 69-86, Rio de Janeiro: Taurus.

MARTINS, I.; COSTA, S.; FREITAS, A. \& PINTO, C., 1991. Aborto induzido em mulheres de baixa renda: Dimensão de um problema. Cadernos de Saúde Pública, 7:251-266.

MATAMALA, M. I., 2000. Mujeres y control social en salud? Hacia una potenciación de género? In: Saúde, Eqüidade e Gênero: Um Desafio para as Políticas Públicas (A. M. Costa, E. MerchánHamann \& D. Tajer, org.), pp. 261-274, Brasília: ABRASCO/Asociación Latinoamericana de Medicina Social/Universidade de Brasília.

MELONI, E., 1999. A medicalização do corpo feminino. In: Questões da Saúde Reprodutiva (K. Giffin \& S. H. Costa, org.), pp. 67-78, Rio de Janeiro: Editora Fiocruz.

MENICUCCI, E. \& VIANNA, L., 1993. Violência conjugal na gravidez. Estudos Feministas, 1:162-165.

MOLINA, A., 1999. Laqueadura tubária: Situação nacional, internacional e efeitos colaterais. In: Questões da Saúde Reprodutiva (K. Giffin \& S. H. Costa, org.), pp. 127-146, Rio de Janeiro: Editora Fiocruz.

MS (Ministério da Saúde), 1995. Plano de Ação para a Redução da Mortalidade Materna. Brasília: MS.

NOLASCO, S., 1988. Identidade Masculina: Um Estudo Sobre o Homem da Classe Média. Dissertação de Mestrado, Rio de Janeiro: Faculdade de Ciências Sociais, Pontifícia Universidade Católica do Rio de Janeiro.

NOLASCO, S., 1995. A desconstrução do masculino: Uma contribuição crítica à análise de gênero. In: A Desconstrução do Masculino (S. Nolasco, org.), pp. 15-29, Rio de Janeiro: Rocco.

PENHA, M. V., 1991. Política e população: Dados sobre um Estado de duas caras. Physis - Revista de Saúde Coletiva, 1:97-115.

PITANGUY, J., 1999. O movimento nacional e internacional de saúde e direitos reprodutivos. In: Questões da Saúde Reprodutiva (K. Giffin \& S. H. Costa, org.), pp. 19-38, Rio de Janeiro: Editora Fiocruz.

RATTNER, D., 1996. Sobre a hipótese de estabilização das taxas de cesárea do Estado de S. Paulo. Revista de Saúde Pública, 30:19-33.

REIS, A. C.; GIFFIN, K. \& SILVEIRA, K., 2001. Mortalidade Feminina no Período Reprodutivo no Brasil e nas Regiões Metropolitanas, 1987 e 1997. Rio de Janeiro: Escola Nacional de Saúde Pública, Fundação Oswaldo Cruz. (mimeo.)

RIBEIRO, M., 1991. Conversando sobre a sexualidade masculina. Revista Brasileira de Sexualidade Humana, 2:35-37.
RNFS (Rede Nacional Feminista de Saúde e Direitos Reprodutivos), 1999. Dossiê. Adolescentes: Saúde Sexual e Reprodutiva. Rio de Janeiro: RNFS.

SAFFIOTI, H., 1992. Rearticulando gênero e classe social. In: Uma Questão de Gênero (A. Costa \& C. Bruschini, org.), pp. 183-215., Rio de Janeiro: Fundação Carlos Chagas/Rosa dos Tempos.

SANTOS, B., 1998. Reinventar a Democracia. Lisboa: Grádiva.

SCOTT, R. P., 1990. O homem na matrifocalidade: Gênero, percepção e experiências do domínio doméstico. Cadernos de Pesquisa, 73:38-47.

SILVA, K.; DÓRSI, E.; LOWNDES, K. \& REIS, A. C. V., 1999. A mortalidade materna no Brasil no período de 1980 a 1993. In: Questões da Saúde Reprodutiva (K. Giffin \& S. H. Costa, org.), pp. 205-226, Rio de Janeiro: Editora Fiocruz.

SILVER, L., 1999. Direito à saúde ou medicalização da mulher. In: Questões da Saúde Reprodutiva (K. Giffin \& S. H. Costa, org.), pp. 299-318, Rio de Janeiro: Editora Fiocruz.

SIMÕES-BARBOSA, R., 2001. Mulheres, Reprodução e AIDS: As Tramas da Ideologia na Atenção à Saúde das Gestantes HIV-Positivas. Tese de Doutorado, Rio de Janeiro: Escola Nacional de Saúde Pública, Fundação Oswaldo Cruz.

SZWARCWALD, C. L. \& LEAL, M. C., 1998. Sobrevivência ameaçada dos jovens brasileiros: A dimensão da mortalidade por armas de fogo. In: Jovens Acontecendo nas Trilhas das Políticas Públicas (Comissão Nacional de População e Desenvolvimento CNPD, org.), pp. 363-393, Brasília: CNPD.

TAJER, D., 2000. Generando la reforma de las reforma. In: Saúde, Eqüidade e Gênero: Um Desafio para as Políticas Públicas (A. M. Costa, E. Merchán-Hamann \& D. Tajer, org.), pp. 145-162, Brasília: ABRASCO/Asociación Latinoamericana de Medicina Social/Universidade de Brasília.

TANAKA, A. C., 2000. Dossiê Mortalidade Materna. Rio de Janeiro: Rede Nacional Feminista de Saúde e Direitos Reprodutivos.

URBANEJA, M., 2000. Género e equidade: Desafios de los procesos de reformas sectoriales de salud. In: Saúde, Eqüidade e Gênero: Um Desafio para as Políticas Públicas (A. M. Costa, E. MerchánHamann \& D. Tajer, org.), pp. 129-144, Brasília: ABRASCO/Asociación Latinoamericana de Medicina Social/Universidade de Brasília.

VALLADARES, D., 1999. Ações de contracepção e assistência ao parto: A experiência do Rio de Janeiro. In: Questões da Saúde Reprodutiva (K. Giffin \& S. Costa, org.), pp. 357-376, Rio de Janeiro: Editora Fiocruz.

VALLADARES, D.; SANCHEZ, S. \& GIFFIN, K., 1987. Mulheres, Participação e Saúde: Uma Experiência. Rio de Janeiro: Facultad Latinoamericana de Ciencias Sociales/Fundo das Nações Unidas para a Infância.

WAWER, M.; LASSNER, K. \& HAUFF, B., 1986. Contraceptive prevalence in the slums of Rio de Janeiro. Studies in Family Planning, 17:44.

Recebido em 24 de abril de 2002

Versão final reapresentada em 15 de agosto de 2002 Aprovado em 12 de setembro de 2002 